\title{
From Protecting a System to Protecting a Global Ecosystem
}

\author{
Moti Yung \\ Google Inc. and Department of Computer Science, Columbia University \\ moti@cs.columbia.edu
}

\begin{abstract}
The area of security used to be classified by technologies: authentication, access control, monitoring, firewalls, cryptography, etc., or by systems: web security, application security, database security, operating systems security, communication security, etc. However, nowadays, in order to operate a service over the Internet, facing customers, one needs to manage a complex infrastructure. This infrastructure hosts many components, numerous technologies, and various devices and computers. The infrastructures and their supported systems are dynamically evolving and can be characterized as ecosystems. On the other hand, attackers over the global Internet exploit weaknesses in one component to attack other parts of the system, taking advantage of lack of global security view. The trend will only increase in the future as more computers are embedded in the infrastructure and more demanding applications are to be developed, and as bigger parts of the global economy move to cyberspace.

Given the state of the art and future developments, it seems mandatory to develop a holistic yet practical approach to protect the computing infrastructure, and to embed it in the ecosystem development process. This infrastructure will be a collection of inter related ecosystems, with providers at the center of each ecosystem. In addition, the ecosystem has to be dynamically evolving. The position stated here is that approaches and methodologies for security will need to change and evolve as well. The view of security as a process rather than as a component is becoming clearer, given the global trend, and the integration of security with the various other steps of the ecosystem evolution is becoming a must as well.
\end{abstract}

\section{Short Biography}

Dr. Moti Yung is a Research Scientist at Google, and an Adjunct Senior Research Faculty at the Computer Science Department, Columbia University. Before that he was a member of IBM Research, was a consultant to leading companies and governments, was with Certco and with RSA Laboratories as well. His main research interests are in the areas of Security, Cryptography and Privacy where he has been working on numerous scientific aspects as well as industrial solutions, for over 25 years. In 2010 he delivered the annual IACR's Distiguished Lecture in Cryptography. His Main research interests are in the areas of security, privacy, cryptography, and their relationships to other areas of engineering and science. 